Notre Dame Journal of Formal Logic

Volume VIII, Number 4, October 1967

\title{
ON THREE RELATED EXTENSIONS OF S4
}

\author{
R. A. BULL
}

1. This paper contains three algebraic exercises which I was led to by some recent discussions with $\mathrm{Mr}$. Geach. The first is to provide a proof of decidability and a characteristic model for the system obtained by extending $S 4^{1}$ with

$$
C M L P C p L p \text {, }
$$

a system originally studied by Geach, and later by various logicians. The others arise from an enquiry of Geach's into systems which can be obtained by extending $\mathrm{S} 4$ with words in a single variable. Could, for example, S4.3 be so presented? It could not, but I was able to establish that its strongest fragment of this form was $\mathrm{S} 4.2+A L C L C p L p L p L C L C L C p L p L p L p$, and that its weakest extension of this form was $\mathrm{S} 4+C M L p A L C p L p L C L C p L p L p$. Geach then pointed out that extending these systems with $C M L p C L C L C p L p L p L p$ gave the trio $54.2+C M L p C L C L C p L p L p L p, \mathrm{D},{ }^{2} \mathrm{~S} 4+C M L p C p L p$ (in order of increasing strength). As it seems to me that this is a more interesting trio than mine, I have adjusted my techniques to apply to it instead. My second exercise, then, is to show that $S 4+C M L p C p L p$ stands to $\mathrm{D}$ as the weakest extension of $\mathrm{S} 4$ with single-variable axioms containing it; my third is to show that the closure in $S 4$ of the single-variables theses of D is $\mathrm{S} 4.2+$ $C M L p C L C L C p L p L p L p$.

I use the usual machinery of closure algebras, the order closure models of [2], and the finite model property. My main concern is with the well-connected closure algebras-i.e. those were

$$
\mathrm{C} a \cap \mathrm{C} b=\wedge \text { iff } a=\wedge \text { or } b=\wedge
$$

which are known to characterize all (normal) extensions of S4, by Lemma 3 of [1]. ${ }^{3}$ I use the symbol - for relative complement, rather than in its normal role of complement proper. Otherwise, anything I use here will be found in [1] or [2], or in the papers referred to there.

2. The system $\mathrm{S} 4+C M L p C p L p$ can also be axiomatised, more conveniently for my purposes, as $\mathrm{S} 4+A L N L P L C p L p$. This new axiom is 
equivalent to $C M L P L C p L p$, which can be seen to imply the old axiom, and be derived from it via $\vdash_{s 4} C M L p M L C p L p, C M L p C p L p$, and $C M L C p L p C C p L p L C p L p$. A well-connected closure algebra verifies $A L N L P L C p L p$ if and only if

$$
\mathrm{C} a=\mathrm{V} \text { or } \mathrm{C} a=a
$$

in it. Thus any sub-set containing $V$ of the elements of such an algebra is closed under $C$. Now suppose that we are given a non-thesis of the system. It must be rejected by some well-connected closure algebra which verifies the system; take the values of its parts in a rejecting evaluation in this algebra and form the (finite) sub-Boolean algebra on them. This is also closed under $C$, so it is a subalgebra of the original algebra; clearly it is a finite well-connected closure algebra which verifies the system and rejects the given non-thesis. Repeating this construction for all non-theses of the system, we have a proof that it has the finite model property.

Since the system has the finite model property it is decidable and is characterized by finite order closure models. It is easily checked that the well-connected finite order closure models which verify the system are those on quasi-ordered sets of the forms represented in Figure 1.

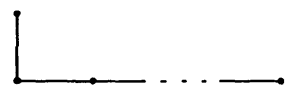

Figure 1.

All these order closure models can be embedded in that on the denumerable quasi-ordered set of the form represented on the left in Figure 1a model which also verifies $A L N L p L C p L p$-so that this is a characteristic model for the system. (That the system lies strictly between D and S5 follows immediately on consideration of their models.)

3. The system $\mathrm{D}$ can be axiomatised as $\mathrm{S} 4+A L C L p L q L C L q L p+$ $A L N L p L C L C L C p L p L p L p$ (given $\vdash_{s 4.3} C M L p L M L p$ and $\vdash \alpha \Longrightarrow \vdash L \alpha$, the latter is equi-derivable with $C M L p C L C L C p L p L p L p)$. Using this axiomatisation it can be established that

(1) $\mathrm{C} a \supseteq \mathrm{C} b$ or $\mathrm{C} a \subseteq \mathrm{C} b$.

(2) If $a \cap b=\wedge$ and $\mathrm{C} a \supseteq \mathrm{C} b$ then $\mathrm{C} a=\mathrm{\vee}$ or $\mathrm{C}(\mathrm{C} a-\mathrm{C} b)=\mathrm{C} a$.

are necessary and sufficient conditions for a well-connected closure algebra to verify $D$. The necessity and sufficiency of (1) for the verification of $A L C L p L q L C L q L p$ are clear. Allocating $\mathrm{C} a-b$ to $p$, we see the necessity of (2) for the verification of $A L N L p L C L C L C p L p L p L p$, for then:

$\mathrm{C} a \supseteq \mathrm{C} a-b \supseteq a$, by the hypothesis that $a \cap b=\wedge$;

$\therefore \mathrm{C}(\mathrm{C} a-b)=\mathrm{C} a$;

$$
\begin{aligned}
\because \mathrm{C}(\mathrm{C}(\mathrm{C} a-b)-(\mathrm{C} a-b)) & =\mathrm{C}(\mathrm{C} a-(\mathrm{C} a-b)) \\
& =\mathrm{C}(\mathrm{C} a \cap b) \\
& =\mathrm{C} b, \text { by the hypothesis that } \mathrm{C} a \supseteq \mathrm{C} b ;
\end{aligned}
$$

$\therefore \mathrm{C} a=\vee$ or $\mathrm{C}(\mathrm{C} a-\mathrm{C} b)=\mathrm{C} a$, by the verification of the word and wellconnectedness. 
Taking $a$ to be the value of $p$ and $b$ to be the value of $C p L p$, the sufficiency of (2) for the verification of $A L N L p L C L C L C p L p L p L p$ is clear.

A well-connected closure algebra which satisfies (1) and (2), and has at most one closed element other than $\wedge$ and $\vee$, verifies $S 4+C M L p C p L p$, for the following reasons. If an element has closure $\wedge$ then it must be $\wedge$. If an element $a$ has a closure which is neither $\wedge$ nor $\vee$ then $\mathrm{C} a$ must be $a$, for otherwise $\mathrm{C}(\mathrm{C} a-a)$ is $\mathrm{C} a$, and (2) is violated when $b$ is taken to be $\mathrm{C} a-a$. Thus all elements satisfy $\mathrm{C} a=\mathrm{V}$ or $\mathrm{C} a=a$, the condition for the algebra to verify $\mathrm{S} 4+C M L p C p L p$. So if a system is not an extension of $\mathrm{S} 4+$ $C M L P C p L p$ then the supposition that it be an extension of $\mathrm{D}$ requires that it be verified by a well-connected algebra satisfying (1) and (2), and having more than one closed element other than $\wedge$ and $V$. I can now show that $\mathrm{S} 4+$ $C M L p C p L p$ is the weakest extension of $\mathrm{S} 4$ with single-variable axioms to contain $\mathrm{D}$, by proving that a single-variable word which is verified by an algebra of this kind is also verified by the order closure model on the quasi-ordered set of Figure 2; for this model

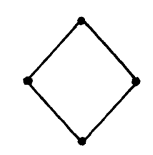

Figure 2 .

does not verify $D$.

Suppose we are given a well-connected closure algebra satisfying conditions (1) and (2), and having elements $C a$ and $C b$, such that $C a \supset C b$ and neither is $\wedge$ or $\vee$. Then the order closure model on the quasi-ordered set of Figure 3 can

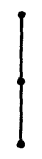

Figure 3 .

be embedded in this algebra by associating the points with $\mathrm{C} b, \mathrm{C} a-\mathrm{C} b$, $\mathrm{V}-\mathrm{C} a$, in descending order. For:

(a) These elements are a disjoint cover of the algebra.

(b) $\mathrm{C}(\mathrm{C} a-\mathrm{C} b)=\mathrm{C} a \supseteq \mathrm{C} b$, by condition (2) and hypothesis.

(c) The algebra verifies $S 4.3$ by condition (1), so it verifies $\vdash s 4.3$ $A L N L p L N L N L p$,

$\therefore \mathrm{C} a=\mathrm{V}$ or $\mathrm{C}(\mathrm{V}-\mathrm{C} a)=\mathrm{V}$, by this verification and well-connectedness; thesis.

$\therefore \mathrm{C}(\mathrm{V}-\mathrm{C} a)=\mathrm{V} \supseteq \mathrm{C} a-\mathrm{C} b$, eliminating the other alternative by hypo-

(d) The converses of these containments do not hold, by hypothesis.

In turn, it may be checked that any subalgebra of the order closure model of Figure 2, generated by the element, can be embedded in the order 
closure model of Figure 3. Therefore every single-variable word which is verified by the given algebra is verified by the order closure model of Figure 2 , as required in the previous paragraph.

4. In this section I show that $S 4.2+C M L p C L C L C p L p L p L p$ contains the single-variable fragment of $D$. Since $D$ is the extension of this system with $A L C L p L q L C L q L p$, it suffices to show that the system contains every word of the form $A L C L \alpha(p) L \beta(p) L C L \beta(p) L \alpha(p)$. This holds if

$$
\text { C } f(a) \supseteq \mathrm{C} g(a) \text { or } \mathrm{C} f(a) \subseteq \mathrm{C}_{g}(a)
$$

in every well-connected closure algebra verifying the system. I prove this linearity by induction on the depth of $C^{\prime} s^{4}$ in $f$ and $g$.

Given a well-connected closure algebra which verifies the system, and an element $a$ of it, I construct sets $A_{n}$ of elements of the algebra such that, with argument $a$, the value of every unary closure-algebraic function having a depth of C's not more then $n$ is in $A_{n}$. These sets are defined inductively in three steps:

(1) $a_{-1}=\mathrm{V}$, $a_{0}=a$, $a_{i}=\mathrm{C} a_{i-1}-a_{i-1}$, for $i \geqslant 1$.

(2) $\alpha_{n}=\left\{a_{n}, a_{n-1}, \mathrm{C} a_{i-1}-\mathrm{C} a_{i} \mid 0 \leqslant i \leqslant n-1\right\}$.

(3) The members of $A_{n}$ are $\wedge$ and the unions of the members of $\alpha_{n}$.

Each $\alpha_{n}$ is a disjoint cover of the algebra, so each $A_{n}$ is closed under the Boolean operators. $A_{0}$ contains $a$; it remains to show that $A_{n+1}$ contains the closures of the members of $A_{n}$. Since the closure of a union of elements equals the union of the closures of the elements, it is sufficient to show that the closure of each member of $\alpha_{n}$ is in $A_{n+1}$.

(a) $\mathrm{C} a_{n}=\left(\mathrm{C} a_{n}-a_{n}\right) \cup a_{n}$

$=a_{n+1} \cup a_{n}$

$\varepsilon A_{n+1}$.

(b) $\mathrm{C} a_{n-1}=\left(\mathrm{C} a_{n}-a_{n}\right) \cup a_{n} \cup\left(\mathrm{C} a_{n-1}-\mathrm{C} a_{n}\right)$, since $\mathrm{C} a_{n-1} \supseteq \mathrm{C} a_{n}$

$=a_{n+1} \cup a_{n} \cup\left(\mathrm{C} a_{n-1}-\mathrm{C} a_{n}\right)$

$\varepsilon A_{n+1}$.

(c) Allocating $a$ to $p$ in $\vdash s 4.2 A L N L p L N L N L p$ and using well-connectedness,

$$
\mathrm{C}\left(\mathrm{C} a_{-1}-\mathrm{C} a_{0}\right)=\wedge \text { or } \mathrm{C}\left(\mathrm{C} a_{-1}-\mathrm{C} a_{0}\right)=\vee
$$

and $\Lambda$ and $\vee$ are in $A_{n+1}$.

(d) Allocating $a_{i-1}$ to $p$ in $A L N L p L C L C L C p L p L p L p$ (this thesis can be derived from $C M L P C L C L C p L P L p L p$ with $\vdash_{44.2} C M L p L M L p$ and $\vdash \alpha \Longrightarrow$ $\vdash L \alpha)$, noting that $C p L p$ takes value $a_{i}$, and using well-connectedness,

$$
\mathrm{C} a_{i-1}=\vee \text { or } \mathrm{C}\left(\mathrm{C} a_{i-1}-\mathrm{C} a_{i}\right)=\mathrm{C} a_{i-1} \text {, for } 1 \leqslant i \leqslant n-1 .
$$


If the first alternative holds, for some $i=m$, the construction may be terminated at $n=m-1$, since then

$$
\mathrm{C} a_{n}=\vee \varepsilon A_{n}
$$

and consideration of $\mathrm{C}\left(\mathrm{C} a_{m-1}-\mathrm{C} a_{m}\right)$ never arises. Therefore we are left with the second alternative:

$$
\begin{aligned}
\mathrm{C}\left(\mathrm{C} a_{i-1}-\mathrm{C} a_{i}\right) & =\mathrm{C} a_{i-1} \\
& =a_{n+1} \cup a_{n} \cup\left(\mathrm{C} a_{n-1}-\mathrm{C} a_{n}\right) \cup \ldots \cup\left(\mathrm{C} a_{i-1}-\mathrm{C} a_{i}\right) \\
& \varepsilon A_{n+1} .
\end{aligned}
$$

Note that the closures of the members of $\alpha_{n}-\vee, \mathrm{C} a_{0}, \mathrm{C} a_{1}, \ldots, \mathrm{C} a_{n}, \wedge$ are linearly ordered under containment, by the definition of the $a_{i}$ 's. It follows that the closures of the members of $A_{n}$, being their unions, are also linearly ordered under containment. The result required in the first paragraph of this section follows immediately, taking $n$ large enough for $A_{n}$ to contain $f(a)$ and $g(a)$.

\section{NOTES}

1. Throughout this paper I take S4, and extensions of it, to be given with $\vdash \alpha \Longrightarrow \vdash L \alpha$ as a derivation rule. (So all systems are automatically normal.)

2. For a brief account of this system of Prior's, and its identification as $\mathrm{S} 4.3+$ $C M L P C L C L C p L p L p L p$, see [1]. I gather that Mr. D. C. McKinson has produced a paper on single-variable words in $D$; I have not seen it.

Added 21-8-65: I learn that this trio has been discussed in Sobocinski's [3], as S4.2.1, S4.3.1, S4.4, and the point made that they are proper extensions of each other.

3. The work of sections 2 and 3 could be done more quickly by using the result, given in a forthcoming paper of mine, that all (normal) extensions of S4.3 are characterized by finite order closure models; however it seems to me that these nuts should be spared the sledge-hammer.

4. The identity function has depth 0 of C's; a Boolean function of functions has the maximum of the depths of C's of those functions for its depth of C's; if $f$ has depth $n$ of C's then C $f$ has depth $n+1$ of C's.

\section{REFERENCES}

[1] Bull, R. A., An algebraic study of Diodorean modal systems. The Journal of Symbolic Logic, vol. 30 (1965), pp. 58-64.

[2] Dummett, M. A. E., and E. J. Lemmon, Modal logics between S4 and S5. Zeitschrift fur mathematische Logik und Grundlagen der Mathematik, bd 5, (1959), pp. 250-264.

[3] Sobociński, B., Modal system S4.4, Notre Dame Journal of Formal Logic, vol. V (1964), pp. 305-312.

Wadham College, Oxford, England 